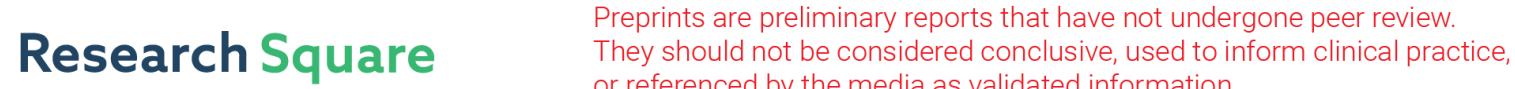 or referenced by the media as validated information. \\ Co-Culture of Mesenchymal Stem Cell Spheres with Hematopoietic Stem Cells Under Hypoxia: A Cost- Effective Method to Maintain Self-Renewal and Homing Marker Expression
}

\section{Fatemeh Amiri}

Hamadan University of Medical Sciences

\section{Ali Asghar Kiani}

Lorestan University of Medical Sciences

Marzie Bahadori

High Institute for Research and Education in Transfusion Medicine mehryar habibi roudkenar ( mhr376@yahoo.com)

Guilan University of Medical Sciences https://orcid.org/0000-0001-8364-7245

\section{Research Article}

Keywords: Mesenchymal stem cell, Sphere, Hematopoietic stem cell, Co-culture, Hypoxia, Endothelial protein $\mathrm{C}$ receptor

Posted Date: August 13th, 2021

DOl: https://doi.org/10.21203/rs.3.rs-801064/v1

License: (c) (1) This work is licensed under a Creative Commons Attribution 4.0 International License. Read Full License

Version of Record: A version of this preprint was published at Molecular Biology Reports on November 6th, 2021. See the published version at https://doi.org/10.1007/s11033-021-06912-x. 


\section{Abstract}

Background: Hematopoietic stem cell (HSC) transplantation is considered a possible treatment option capable of curing various diseases. The aim of this study was the co-culturing of mesenchymal stem cell (MSC) spheres with HSCs under hypoxic condition to enhance the proliferation, self-renewal, stemness, and homing capacities of HSCs.

Methods and results: HSCs were expanded after being subjected to different conditions including cytokines without feeder (Cyto), co-culturing with adherent MSCs (MSC), co-culturing with adherent MSCs+ hypoxia (MSC+ Hyp), co-culturing with MSCs spheres (Sph-MSC), co-culturing with MSCs spheres+ hypoxia (Sph-MSC+ Hyp), co-culturing with MSC spheres+ cytokines (Sph-MSC+Cyto). After 10 days, total nucleated cell (TNC) and $\mathrm{CD} 34^{+} / \mathrm{CD}^{-} 8^{-}$cell counts, colony-forming unit assay (CFU), long-term culture initiating cell (LTC-IC), the expression of endothelial protein $C$ receptor (EPCR), nucleostemin (NS), nuclear factor I/X (NfIX) CXCR4, and VLA-4 were evaluated. The TNC, CD34 ${ }^{+} / \mathrm{CD} 38^{-}$cell count, CFU, and LTC-IC were higher in the Sph-MSC+ Hyp and Sph-MSC+ Cyto groups as compared with those of the MSC+ Hyp group $(P<0.001)$. The expanded HSCs co-cultured with MSC spheres in combination with hypoxia expressed more EPCR, CXCR4, VLA-4, NS, and Nfix mRNA. The protein expression was also more up-regulated in the Sph-MSC+Cyto and Sph-MSC+ Hyp groups.

Conclusion: Co-culturing HSCs with MSC spheres under hypoxic condition not only leads to higher cellular yield but also increases the expression of self-renewal and homing genes. Therefore, we suggest this approach as a simple and non-expensive strategy that might improve the transplantation efficiency of HSCs.

\section{Introduction}

Hematopoietic stem cells (HSCs) are a heterogeneous population of stem cells that have stemness and self-renewal capability and can differentiate to all blood cell lineages [1-3]. Based on their self-renewal potentialities, HSCs are mainly divided into two types, long-term HSCs and short-term HSCs [3-5]. The transplantation of HSCs is considered a curative and/or supportive treatment for several hematologic and non-hematologic diseases [6-8]. However, transplantation of short-term HSCs instead of long-term HSCs, and the poor homing rate are known as main obstacles of HSC transplantation [7, 9-11].

So far, various studies have been conducted to address some of the mentioned obstacles [3, 12]. In vitro methods use different cytokine cocktails to expand HSCs, which might be an expensive approach. In recent decades, co-culturing mesenchymal stem cells (MSCs) with HSCs has been employed for harvesting more numbers of HSCs $[13,14]$.

MSCs are potent valuable cells that can be isolated from bone marrow, umbilical cord blood, umbilical cord tissue and, etc. [13-15]. MSCs and their derivatives are used in gene therapy and cell therapy for treating a variety of diseases [16-18]. On the other hand, MSCs have the potentiality to secrete stem cell factor (SCF), granulocyte monocyte colony-stimulating factor (GM-CSF), thrombopoietin (TPO), 
angiopoietins (Angs), Fms-related tyrosine kinase 3-Ligand (Flt3-L), and wingless-related integration site (Wnts) that are involved in HSCs proliferation, self-renewal, and stemness [4, 19-22]. MSC-secreted SCF has positive effects on the proliferation, survival, and self renewality of HSCs [4]. Angs enhance the symmetric division of HSCs and preserve their stemness capacity [19, 23]. Wnts and their related signaling pathways play critical roles in the fate of stem cells including HSCs [24, 25]. Some of the applied methods for enhancing the expansion of HSCs are high-tech but they are costly as well.

According to our present study, reinforcement of MSC paracrine effects through the implementation of co-culturing MSCs with HSCs seems a feasible strategy to reach more numbers of potent engraftable cells. Culturing in the non-adherent phase that allows for the formation of spheres is one of the practical methods utilized for improving the differentiation and secretory capacities of MSCs [15, 20, 26, 27]. Suspension cultivation of MSCs somehow primes them to show more supportive effects on HSCs [28, 29]. Moreover, monolayer culturing of MSCs leads to diminished proliferation and secretory potentialities along with induced cellular senescence [30].

Based on our previous experience, this study aims to expand HSCs in a co-culturing system containing MSC spheres under hypoxia condition for improving their self-renewality and homing capacities. Our results demonstrated that co-culturing MSCs spheres with HSCs under hypoxic condition increases the number of HSCs and results in an increased colony-forming unit assay (CFU-assay) and long-term culture initiating cell (LTC-IC) capacities. The expanded HSCs demonstrated more rates of endothelial protein C receptor (EPCR), nucleostemin (NS), nuclear factor I/X (Nfix) expression indicating that these cells exhibit superior self-renewal capability and stemness ability than those of HSCs co-cultured with monolayer adherent MSCs under the same oxygen pressure. The up-regulated expression of $\mathrm{C}-\mathrm{X}$-C chemokine receptor type 4 (CXCR4) and very late antigen (VLA-4) in HSCs co-cultured with MSCs spheres proved their homing potentiality that might result in enhanced engraftment rate after transplantation.

\section{Material And Methods}

\section{Isolation of Wharton Jelly (WJ)-MSC spheres and evaluation of their growth factor secretion capability}

WJ-MSCs were subjected to suspension culturing conditions to form WJ-MSC spheres as described in our previous study [15]. Briefly, the WJ-MSCs were separated from fresh umbilical cord samples after the necessary consent forms were filed by parents. The WJ-MSCs were seeded in Poly-HEMA-coated plates and incubated under hypoxic condition containing $5 \% \mathrm{O}_{2}$ (New Brunswick, Germany) to support the forming of spheres. Next, the formed spheres were cultured in serum-free media for 48 hours. The collected supernatant was concentrated using Vivaspin ${ }^{\circledR} 500$ (Sartorius, Germany) with centrifugal force. Concentrations of growth factors including Angs, Wnts, SCF, and Flt3-L were measured using ELISA method (MyBioSource, USA) according to the manufacturer's protocol. 


\section{Isolation and cultivation of HSCs}

After the necessary consent forms were filed, HSCs were isolated from fresh umbilical cord blood using ficoll gradient protocol as described previously [31]. CD34 ${ }^{+}$cells were positively selected using magneticactivated cell sorting (MACS) according to the kit instructions (Miltenyi Biotec, Germany). The purity of CD34 ${ }^{+}$cells and their CD38, CD90, and CD45RA expression levels were analyzed using flow cytometry (Life Technologies Attune Nxtm, USA). The used antibodies and fluorochromes are as follows: CD34 APC/PE, CD38 FITC, CD45RA PE, and CD90 FITC (BD, Biosciences, Germany). The cells were mixed with serum-free stem span media (Stem Cell Technologies, USA) containing a cytokine cocktail (Flt3-L, TPO, and SCF) and then were seeded on culture plates. The culture medium was refreshed twice per week. This group of HSCs (expanded in cytokines without feeder layer condition) was named "Cyto".

\section{Co-culturing HSCs with adherent MSCs or MSC spheres under hypoxia or normoxia}

MSC spheres were transferred into culture plates and the mixture of HSCs with stem span medium containing cytokines (named Sph-MSC+ Cyto) or without cytokines (named Sph-MSC) were added to the plates. Co-culturing HSCs with adherent monolayer MSCs (named MSC) was considered as another experimental group. All of the mentioned culture plates were incubated at $37^{\circ} \mathrm{C}$ for 10 days $\left(20 \% \mathrm{O}_{2}\right.$ and $5 \% \mathrm{CO}_{2}$ ). The plates containing MSC spheres with HSCs without cytokine addition and the plates containing adherent MSC with HSCs incubated under reduced $\mathrm{O}_{2}$ pressure $\left(5 \% \mathrm{O}_{2}\right)$ to induce hypoxia (named Sph-MSC+ Hyp and MSC+ Hyp, respectively) were considered as other experimental groups. The culture medium was exchanged twice per week. After 10 days, MSC spheres were separated from HSCs using filters and TNC counting was performed using trypan blue staining and hemocytometer. Then the number of $\mathrm{CD} 4^{+} / \mathrm{CD} 38^{-}$cells was also calculated based on the TNC counting and their defined percentage by flow cytometry. The fold change in $\mathrm{TNC}$ and $\mathrm{CD} 34^{+} / \mathrm{CD} 38^{-}$cell number was calculated by comparing with the initial number of the seeded cells.

\section{CFU-assay}

On the $10^{\text {th }}$ day of the experiment, the expanded cells of Cyto, MSC, MSC+ Hyp, Sph-MSC, Sph-MSC+ Hyp, and Sph-MSC+ Cyto groups were cultivated in iscove's MDM medium (Sigma, Germany) containing $1 \%$ methylcellulose, and they were incubated for 14 days. Clusters containing 50 or more cells were counted to calculate CFU capacity. Granulocyte macrophage-colony forming units (CFU-GM), erythroid burst/colony-forming units (BFU-E/CFU-E), and granulocyte erythrocyte monocyte megakaryocyte-colony forming units (CFU-GEMM) were scored and counted in triplicate by laboratory experts. The CFU fold change of expanded cells was estimated by comparing it with the CFU of fresh unexpanded cells. 


\section{LTC-IC-assay}

LTC-IC assay was conducted on an irradiated feeder. The expanded CD $34^{+}$cells were seeded in MyelocultMyelocM5300 (Stem Cell Technologies, USA) and the medium was changed twice a week. After 6 weeks, the viability of harvested cells was determined using trypan blue exclusion dye, and two thousand cells were subjected to CFU-assay according to the previously mentioned procedure. The enumerated colonies were considered as LTC-IC. The LTC-IC fold change of the expanded cells was estimated in comparison to the LTC-IC of fresh unexpanded cells.

\section{Real-time reverse transcription polymerase chain reaction (qRT-PCR)}

qRT-PCR was performed to measure the expression levels of $N S$ and $N f i x$ as self-renewal genes as well as CXCR4 and VLA-4 as homing genes using Syber green dye. Briefly, $6 \mu \mathrm{l}$ of Syber green master mix (Takara, Japan), $1 \mu$ l of synthesized cDNA (BIONEER, South Korea), and $10 \mu \mathrm{mol} / \mathrm{l}$ of specific primers were mixed and the total volume was adjusted to $12 \mu \mathrm{l}$. The primer sequences were as follows: $C X C R 4$ F: 5'-CTATTGAACCCCATCCTGCT-3' R: 5'- TCCACGATGAATGCTCGCTTT-3', VLA-4 F: 5'ATGTTGCGCATGTTCTACTG -3' R: 5'-AGCCTTCCACATAACATATGAG-3', NSF: 5'CTGATGCTGGAGTTGGATGC -3' R: 5'- CATTGATCACGTTGAAGGC-3', Nfix F: 5'CATTCTTGTCGCCGTCCTG-3' R: 5'-TCCGTTCCGCAAGCATCAC-3' and B-actin F: 5'GATACCGCAAATGTGACACG-3' R: 5'-GGGCTCACAGGAACAGTTCT-3'. qRT-PCR was performed using the LightCycler® 96 System (Roche, Bavaria, Germany) in triplicate. The specificity of PCR amplification was analyzed by melting curve. The relative expression of the mentioned genes in comparison with the expression of $\beta$-actin was determined using the $2^{(-\Delta \Delta C T)}$ method. PCR conditions were as follows: initial denaturation at $95^{\circ} \mathrm{C}$ for $15 \mathrm{~min}$ followed by 40 cycles of 20 s at $95^{\circ} \mathrm{C}, 60$ s at $60^{\circ} \mathrm{C}$ for $V L A-4, N S, N f i x$, and $\beta$-actin, and $63^{\circ} \mathrm{C}$ for $C X C R 4$.

\section{Immunophenotyping for EPCR, CXCR4, and VLA-4 expression}

After 10 days of HSCs expansion, the expression of EPCR, CXCR4, and VLA-4 was assayed using flow cytometer (Life Technologies Attune Nxtm, USA). The cells were incubated and stained with an optimized amount of related antibodies at room temperature for 30 minutes in the dark. Then, the cells were checked by the flow cytometer instrument.

\section{Western blot analysis}


Total protein of expanded HSCs was extracted using lysis buffer (Roche, Germany). After electrophoresis of the protein samples on $12 \%$ SDS-polyacrylamide gel, they were transblotted onto polyvinylidene fluoride membrane (Roche, Germany) using semi-dry blotter (Bio-Rad, USA) in an optimized voltage for an appropriate duration. Then, the membranes were incubated with anti-NS (Abcam, UK), anti-Nfix (Merck, Germany) and anti- $\beta$-actin (Sigma, USA) antibodies at optimized conditions (for anti-NS antibody: 1:3000 dilution, 3 hours at room temperature with shaking, for anti-Nfix antibody: 1:5000 dilution, 3 hours at room temperature with shaking, and for anti- $\beta$-actin antibody: 1:2000, 2 hours at room temperature with shaking). After immersing the membranes in the diluted (1: 1000) secondary antibody (Abcam, UK), ECL substrate solution was poured on the membranes. Furthermore, images were captured using a gel doc imager (Bio-Rad, USA) and the protein expression levels were semi-quantified using Image Lab software.

\section{Statistical analysis}

Parametric ANOVA test was used for data analysis and the $\mathrm{p}$-value of $\mathrm{P}<0.05$ was considered significant. The experiments were carried out in duplicate in 3 independent experiments.

\section{Results}

\section{WJ-MSCs form spheres with higher SCF, Wnts, Angs, and Flt3-L secretion ability under hypoxic condition}

WJ-MSCs were isolated successfully in both adherent (Fig. 1a) and non-adherent spheroid form (Fig. 1b). As shown in Fig. 1c, cultivation of WJ-MSCs in Poly HEMA-coated plates for suspension culturing under hypoxic condition enhanced their paracrine effect especially in the case of Flt-3 $(P<0.001)$. The concentrations of SCF, Wnts, and Angs were more in the conditioned medium of MSC spheres in comparison to those of the conditioned medium of adherent MSCs $(P<0.001)$ (Fig. 1c).

\section{HSCs show higher proliferation rate while co-cultured with MSC spheres under hypoxia condition}

MACS was used to separate cells expressing CD34 and CD90 (Fig. 2all) and not expressing CD38 and CD45RA (Fig. 2allI). These cells expressed very a low level of EPCR (Fig. 2alV). HSCs were expanded in different conditions including cytokines without feeder (Cyto), co-culturing with adherent MSCs (MSC), co-culturing with adherent MSCs + hypoxia (MSC + Hyp), co-culturing with MSCs spheres (Sph-MSC), coculturing with MSCs spheres + hypoxia (Sph-MSC + Hyp), and co-culturing with MSC spheres + cytokines (Sph-MSC + Cyto). Figure 2b and Fig. 2c represent MSC + Hyp and Sph-MSC + Hyp groups after 10 days, respectively. 
According to the cell enumeration results, the count and fold increase of TNC along with $\mathrm{CD} 34^{+} / \mathrm{CD} 38^{-}$ cells were significantly higher $(P<0.001)$ in Sph-MSC + Hyp and Sphe-MSC + Cyto than those of the MSC and MSC + Hyp groups co-cultured with adherent MSCs (Table 1). The fold expansion average of TNC for Cyto, MSC, MSC + Hyp, Sph-MSC, Sph-MSC + Hyp, and Sph-MSC + Cyto groups were 32.8 $\pm 2.9,27.9 \pm 1.1$, $32.95 \pm 2.7,47.9 \pm 2.7,54.3 \pm 3.3$, and $59.1 \pm 2.9$, respectively. We observed a $48.9 \pm 2.5$-fold increase in $\mathrm{CD} 4^{+} / \mathrm{CD}^{-} 8^{-}$cells in the Sph-MSC + Hyp group. The mentioned parameters did not significantly differ in the Sph-MSC + Cyto group as compared to the Sph-MSC + Hyp group (Table 1). The fold change of TNC and $\mathrm{CD}_{34}{ }^{+} / \mathrm{CD}_{3} 8^{-}$cells was calculated in comparison with the initial number of seeded cells.

Table 1

$\mathrm{CD} 4^{+} / \mathrm{CD} 38^{-}$and total nucleated cell count and fold change at 10 th day of expansion under different conditions.

\begin{tabular}{|c|c|c|c|c|}
\hline \multirow[t]{2}{*}{ Culture condition } & \multirow{2}{*}{$\begin{array}{l}\text { CD } 34^{+} / C D 38^{-} \times 10^{5} \\
\text { Count }\end{array}$} & \multirow{2}{*}{$\begin{array}{l}\text { CD34 }{ }^{+} / C^{-} 38^{-} \\
\text {Fold change }\end{array}$} & \multirow{2}{*}{$\begin{array}{l}\text { TNC } \times 10^{5} \\
\text { Count }\end{array}$} & \multirow{2}{*}{$\begin{array}{l}\text { TNC } \\
\text { Fold change }\end{array}$} \\
\hline & & & & \\
\hline Cyto & $3.13 \pm 0.95$ & $31.59 \pm 2.2$ & $3.25 \pm 0.92$ & $32.8 \pm 2.9$ \\
\hline MSC & $2.49 \pm 0.71$ & $25.3 \pm 2.9$ & $2.83 \pm 0.79$ & $27.9 \pm 1.1$ \\
\hline MSC + Hyp & $3.5 \pm 0.83$ & $34.1 \pm 2.9$ & $3.33 \pm 0.96$ & $32.95 \pm 2.7$ \\
\hline Sph-MSC & $5.4 \pm 0.78$ & $39.15 \pm 1.7$ & $5.95 \pm 1.05$ & $47.9 \pm 2.7$ \\
\hline Sph-MSC + Hyp & $6.16 \pm 1.19^{\star \star \star}$ & $48.9 \pm 2.5^{\star \star \star}$ & $7.09 \pm 1.23^{\star \star \star}$ & $54.3 \pm 3.3^{\star \star \star}$ \\
\hline Sph-MSC + Cyto & $7.1 \pm 1.59^{\star \star \star}$ & $50.5 \pm 3.1^{\star \star \star}$ & $7.53 \pm 1.61^{\star \star \star}$ & $59.1 \pm 2.9^{\star \star \star}$ \\
\hline \multicolumn{5}{|c|}{$\begin{array}{l}\text { TNC: Total nucleated cell count, Cyto: Cytokines without feeder, MSC: Co-culturing with adherent } \\
\text { MSCs, MSC + Hyp: Co-culturing with adherent MSCs under hypoxic condition, Sph-MSC: Co-culturing } \\
\text { with MSC spheres, Sph-MSC + Hyp: Co-culturing with MSC spheres under hypoxic condition, Sph-MSC } \\
+ \text { Cyto: Co-culturing with MSC spheres with cytokine addition, ***P }<0.001 \text { Sph-MSC + Cyto and Sph- } \\
\text { MSC + Hyp vs MSC and MSC + Hyp. The fold change was calculated vs initial seeded cells. }\end{array}$} \\
\hline
\end{tabular}

\section{HSCs co-cultured with MSC spheres show more enhanced CFU and LTC-IC capacitiesthan those of HSCs co-cultured with adherent MSCs}

CFU assay and LTC-IC were performed to evaluate the clonogenicity of HSCs. According to the results presented in Fig. 3al, all and alll, the expanded cells could form CFU-GM, BFU-E/CFU-E, and CFU-GEMM. We observed more clonogenic capacity in Sph-MSC + Hyp and Sph-MSC + Cyto in comparison to that of the MSC + Hyp group $(\mathrm{P}<0.001)$ (Fig. 3b). The CFU fold increase numbers in Sph-MSC + Hyp vs MSC + Hyp were determined as follows: a 1.9-fold increase in total CFU, a 1.7-fold increase of CFU-GM, a 1.6-fold increase of BFU-E/CFU-E, and a 2.1-fold increase of CFU-GEMM. The fold change of different colonies in 
Sph-MSC + Cyto was not meaningfully different from that of Sph-MSC + Hyp except for CFU-GEMM $(P<0$. 05) (Fig. 3b).

The results of the LTC-IC assay indicated a higher fold increase in long-term HSCs co-cultured with MSC spheres both with or without the addition of cytokines $(P<0.01)$. On the other hand, Sphe-MSC + Cyto and Sph-MSC + Hyp had more improvement in LTC-IC capacity in comparison to that of MSC + Hyp $(P<0.01)$ (Fig. 3c). The fold change of CFU and LTC-IC was calculated in comparison with fresh unexpanded cells.

\section{Sph-MSC + Cyto and Sph-MSC + Hyp show more up- regulated expression of self-renewal and homing genes}

NS and Nfix along with CXCR4 and VLA-4 expression were quantified using qRT-PCR. The up-regulation of $N S, N f i x, C X C R 4$, and VLA-4 expression was detected (Fig. 4a). HSCs expansion in presence of MSC spheres and hypoxia led to enhanced expression of self-renewal and homing genes especially in terms of NfIX and CXCR4 (P<0.001) (Fig. 4a). The expression of NS, NfIX, CXCR4, and VLA-4 in Sph-MSC + Hyp in comparison to MSC + Hyp was significantly up-regulated $(\mathrm{P}<0.001)$. The different expression levels of $N S, C X C R 4$, and VLA-4 were not significant in Sph-MSC + Cyto vs Sph-MSC + Hyp. However, a more upregulated expression of Nfix was detected in Sph-MSC + Cyto $(\mathrm{P}<0.05)$ (Fig. 4a).

\section{The Expression of NS and Nfix proteins are elevated in HSCs co-cultured with MSC spheres}

Western blot was performed to confirm the expression of NS and Nfix proteins. The sample image of protein bands is presented in Fig. 4b. The protein density was semi-quantified. The increased amount of protein levels was detected in Sph-MSC + Cyto and Sph-MSC + Hyp, not only in terms of NS but also in terms of Nfix as compared with those of MSC + Hyp $(P<0.001, P<0.01)$ (Fig. 4c).

\section{MSCs spheres induce a higher level of EPCR, CXCR4, and VLA-4 in the expanded HSCs}

Co-culturing HSCs with MSC spheres under hypoxia conditions resulted in a higher level of EPCR expression in comparison with that of HSCs co-cultured with adherent MSCs under hypoxia. Figure 5al and 5all represent flow cytometry graphs related to the EPCR expression in CD34 positive cells in the MSC + Hyp and Sph-MSC + Hyp groups, respectively. The percentage of EPCR expression was quantified in all of the experimental groups. As shown in Fig. 5b, Sph-MSC + Hyp had the most EPCR expression level $(P<0.001)$. Furthermore, the CXCR4 surface marker was expressed about $50 \%$ in Sph-MSC + Hyp that was higher than its expression in MSC + Hyp (21.08\%) (Fig. $5 \mathrm{cl}$ and cll). Quantification of CXCR4 expression revealed that Sph-MSC + Hyp and Sphe-MSC + Cyto expressed more CXCR4 (Fig. 5d) $(P<$ 
0.001). Sph-MSC + Hyp also expressed VLA-4 on their surface even higher than Sphe-MSC + Cyto (Fig. 5el, 5ell, and 5f). As demonstrated in Fig. 5f, hypoxia and co-culturing with MSC spheres induced a higher level of VLA-4 expression $(P<0.01)$.

These results proved that MSC spheres harbor superior hematoprotective potentialities in comparison with those of adherent MSCs. The results of co-culturing with MSC spheres without cytokine addition along with hypoxia induction are comparable to those of culturing under cytokine addition. MSC spherebased co-culturing with or without cytokine addition showed similar supportive effects on proliferation and self-renewal/homing marker expression of HSCs.

\section{Discussion}

Improving the surrounding microenvironment of HSCs is critical for their expansion and obtaining an adequate number of cells ex vivo. Co-culturing with MSCs is one of the applicable methods for the expansion of HSCs $[13,14]$. Following our previous experience in increasing the supportive effects of MSCs on HSCs expansion [31], in this study, we examined the impacts of different co-culturing systems on the expansion of HSCs. WJ-MSCs were used as a feeder layer. Advantages of birth-associated tissuederived MSCs have been described previously $[14,15]$. The spheroid form of MSCs was used for coculturing with HSCs along with hypoxia. We previously reported the improvement of plasticity and differentiation potentialities of MSCs under suspension cultivation [15, 32]. Moreover, the paracrine effects of MSCs improve under spheroid conditions [20,26, 27, 33]. Scientific evidence confirms the positive effects of hypoxia on the therapeutic applications of MSCs [34-36].

Our results indicated elevated levels of Angs, Wnts, SCF, and Flt3-L in the conditioned medium of MSC spheres cultivated under hypoxic condition. MSCs secrete Angs which is involved in the process of regeneration [37]. Not only it influences the niche and fate of HSCs in vivo, but it is also involved in their survival and stemness ex vivo [19, 23, 37]. Flt3-L and SCF are well-known crucial growth factors for the proliferation, survival, and self-renewal of HSCs $[4,21]$. Activation of Wnts and its related signaling pathways, especially the $\beta$-catenin-dependent pathway, improves the proliferation of HSCs and consequently increases the number of long-term HSCs [24, 25].

Co-culturing MSC spheres and HSCs led to an increased number of expanded CD34 ${ }^{+} / \mathrm{CD}^{-} 8^{-}$cells. The percentage of expanded $\mathrm{CD} 34^{+}$cells and the duration of expansion are the variable parameters in the reported studies. Different expansion conditions, various defined times for CD34 evaluation, and interchangeability of the $\mathrm{CD} 34^{+}$and $\mathrm{CD} 34^{-}$populations $[3,38]$ might lead to different results.

In 2017, Ahmadinejad et al. expanded HSCs under different conditions including co-culturing with MSCs, co-culturing with MSCs + cytokines, and cytokines only. In the mentioned study, a maximum increase in the number of $\mathrm{CD}_{3} 4^{+}$cells was observed when HSCs were co-cultured with MSCs + cytokines after 7 days. They concluded that the high-level expression of DNA methyltransferase 1 was the mechanism underlying this event [39]. Our results showed the increased fold change of CFU and LTC-IC. Co-culturing 
of MSC spheres with HSCs under hypoxia led to an enhanced amount of Angs, Wnts, SCF, and Flt3-L which resulted in an improved level of clonogenicity along with long-term cells in the expanded cells. In 2014, Oubari et al. assayed CFU and LTC-IC capacity of HSCs in expansion conditions, cytokine cocktail containing SCF, TPO, and Flt3-L, placenta-derived MSC feeder layer, and placenta-derived MSC layer with Flt3-L. Their results indicated that using placenta-derived MSCs only in the presence of Flt3-L led to an increased LTC-IC along with an enhanced CFU capacity which was similar to the effects of using cytokine cocktail without the presence of any feeder layer. They emphasized the crucial role of Flt3-L in HSCs expansion in vitro [22].

In our study, an increased expression level of endothelial protein C receptor (EPCR) was detected by flow cytometry in co-culturing of HSCs with MSC spheres. EPCR is a potential long-term engraftment marker of HSCs [40, 41]. The subgroup of CD34 + CD 90 + CD45- cord blood HSCs express EPCR [41]. EPCR and its related signaling pathways control the recruitment of long-term HSCs by decreasing nitric oxide production and increasing the affinity of the VLA-4 homing marker [42]. The paracrine effects of MSC spheres particularly by Wnts maintain the stemness and long-term capability of EPCR positive HSCs [25] and cause a slow cell cycling state [43]. In 2017, Fares et al. studied the effects of UM171, as an agonist of HSCs, on the proliferation rate of HSCs ex vivo and their reconstitution ability in vivo. Their study reported that UM171 caused an increased expression of EPCR among long-term HSCs [42]. Furthermore, various other chemical components have been used to induce EPCR expression and to harvest more potent primitive HSCs [44].

Expansion of HSCs in co-culture with MSC spheres up-regulated genes involved in the survival, selfrenewality, and homing of HSCs. NS influences different physiologic and pathologic processes since it can coordinate cell self-renewal [45]. It has effects on cell proliferation without any interference with the differentiation behavior of the cells [46]. Nfix plays an essential role in the survival of primitive HSCs by elevating the expression of $c-M p l$, reducing apoptosis, and protecting the cells against stressful conditions [47, 48]. In 2013, Holmfeldt and colleagues knocked down the Nfix gene in hematopoietic stem progenitor cells (HSPCs). The Nfix-depleted HSPCs lost their clonogenicity due to the down-regulation of survival genes such as Erg, Mecom, and Mpl. The mice receiving the Nfix-depleted HSPCs showed poor hematopoietic repopulation because of the increased number of apoptotic cells in the bone marrow [48]. In 2018, Hall et al. over-expressed Nfix in HSCs and expanded them in different conditions. They extended the period of expansion from 20 to 40 days [47]. In a study, expression of NS, as a stemness monitoring marker, was measured in different sub-fractions of primitive HSCs such as CD150 / CD 48 $/$ CD $34^{-}$and $\mathrm{CD} 150^{+} / \mathrm{CD}^{-} 8^{-} / \mathrm{CD} 4^{+} \mathrm{LT}$-HSCs. This marker was expressed in both of the abovementioned subfractions [2].

CXCR4/SDF-1 axis is fundamental for homing and trafficking of any type of stem cells $[38,49]$. On the other hand, the expression of VLA-4 is critical for HSCs mobilization and homing [22, 38]. In 2018, Mousavi et al. expanded HSCs in three-dimensional nanofiber scaffolds. The expression of homing genes such as VLA-4 and CXCR4 was evaluated. Consistent with our results, they reported an increased fold change for the expression of CXCR4 and VLA-4 [38]. In another study, the impact of culturing HSCs on the 
expression of CD49d, a VLA-4 subunit, with or without feeder layer with using different cytokine cocktails was reported and it was concluded that the combination of MSC feeder layer and Flt-3 addition induced high levels of VLA-4 surface expression in HSCs [22].

In 2016, Huang et al. designed a three-dimensional co-culturing system using bone-made scaffolds. These researchers mixed HSCs with MSCs or/and osteoblasts and seeded them on the scaffolds. CFU and LTC-IC capacities were reinforced in HSCs co-cultured with MSCs and osteoblasts. A high engraftment rate was confirmed after transplantation of these HSCs to immunodeficient mice [28]. In 2015, Kadekar et al. compared the supportive effects of placenta-derived and umbilical cord-derived MSC feeder on HSCs. They reported superior supportive effects of placenta-derived MSC feeder on HSCs especially in terms of proliferation and CFU ability [14]. Culturing MSCs on Polydimethylsiloxane polymer led to enhanced secretion of Ang-1 and TPO. Co-culturing these MSCs with HSCs increases the myeloid differentiation capacity of HSCs and induces CD123 expression [26]. According to in vitro studies, expanding HSCs on the MSC-feeder layer in combination with culturing under hypoxia conditions results in an increased number of $\mathrm{CD} 34^{+}$cells, enhanced clonogenicity, and improved homing and migration capacities [34, 35].

\section{Conclusion}

Among different designed HSC expansion conditions, co-culturing with MSC spheres showed more hematoprotective potentialities. MSC spheres co-cultured under hypoxia condition showed supportive effects on proliferation, self-renewal, and homing capability in the majority of HSCs which was similar to those of using as cytokine addition condition. Using an MSC sphere-based co-culture system under hypoxia condition might be a potent and non-expensive alternative for cytokine cocktails that are usually used for the expansion of HSCs.

\section{Declarations}

\section{Funding}

This work was supported by the Hamadan University of Medical Sciences [grant number: 990126370]; and the Guilan University of Medical Sciences [grant number: IR.GUMS.REC.1396.508].

\section{Conflict of interest}

The authors declare that they have no conflict of interest.

\section{Author contributions}


Conceptualization: [Fatemeh Amiri and Mehryar Habibi Roudkenar], Methodology: [Fatemeh Amiri, Ali Asghar Kiani and Marzyie Bahadori], , Formal analysis: [Fatemeh Amiri and Mehryar Habibi Roudkenar], Investigation and Writing - original draft preparation: [Fatemeh Amiri], Review and editing: [Marzyie Bahadori and Mehryar Habibi Roudkenar], Funding acquisition: [Fatemeh Amiri and Mehryar Habibi Roudkenar and Mehryar Habibi Roudkenar], Supervision: [ Mehryar Habibi Roudkenar].

\section{Acknowledgement}

This work was supported by the Hamadan University of Medical Sciences [grant number: 990126370]; and the Guilan University of Medical Sciences [grant number: IR.GUMS.REC.1396.508]. All experimental procedures were approved by Hamadan University of Medical Sciences [NO: IR.UMSHA.REC.1399.035].

\section{References}

1. Saleh M (2015) The impact of mesenchymal stem cells on differentiation of hematopoietic stem cells. Advanced pharmaceutical bulletin 5(3):299

2. Ali MA, Fuse K, Tadokoro Y, Hoshii T, Ueno M, Kobayashi M et al (2017) Functional dissection of hematopoietic stem cell populations with a stemness-monitoring system based on NS-GFP transgene expression. Scientific reports 7(1):1-12

3. Kita K, Xiu F, Jeschke MG (2014) Ex vivo expansion of hematopoietic stem and progenitor cells: Recent advances. World Journal of Hematology 3(2):18-28

4. Aqmasheh S (2017) Effects of mesenchymal stem cell derivatives on hematopoiesis and hematopoietic stem cells. Advanced pharmaceutical bulletin 7(2):165

5. Copelan EA (2006) Hematopoietic stem-cell transplantation. N Engl J Med 354(17):1813-1826

6. Bazinet A, Popradi G (2019) A general practitioner's guide to hematopoietic stem-cell transplantation. Current Oncology 26(3):187

7. Duarte RF, Labopin M, Bader P, Basak GW, Bonini C, Chabannon C et al (2019) Indications for haematopoietic stem cell transplantation for haematological diseases, solid tumours and immune disorders: current practice in Europe, 2019. Bone marrow transplantation 54(10):1525-1552

8. Takagaki S, Yamashita R, Hashimoto N, Sugihara K, Kanari K, Tabata K et al (2019) Galactosyl carbohydrate residues on hematopoietic stem/progenitor cells are essential for homing and engraftment to the bone marrow. Scientific reports 9(1):1-11

9. Merli P, Caruana I, De Vito R, Strocchio L, Weber G, Del Bufalo F et al (2019) Role of interferon-y in immune-mediated graft failure after allogeneic hematopoietic stem cell transplantation. haematologica 104(11):2314-2323

10. Caocci G, Greco M, La Nasa G (2017) Bone marrow homing and engraftment defects of human hematopoietic stem and progenitor cells. Mediterranean journal of hematology and infectious diseases, $9(1)$ 
11. Ozdemir ZN, Bozdağ SC (2018) Graft failure after allogeneic hematopoietic stem cell transplantation. Transfus Apheres Sci 57(2):163-167

12. Zhang Y, Shen B, Guan X, Qin M, Ren Z, Ma Y et al (2019) Safety and efficacy of ex vivo expanded CD34 + stem cells in murine and primate models. Stem Cell Res Ther 10(1):173

13. Matsuoka Y, Nakatsuka R, Sumide K, Kawamura H, Takahashi M, Fujioka T et al (2015) Prospectively Isolated Human Bone Marrow Cell-Derived MSC s Support Primitive Human CD 34-Negative Hematopoietic Stem Cells. Stem Cells 33(5):1554-1565

14. Kadekar D, Kale V, Limaye L (2015) Differential ability of MSCs isolated from placenta and cord as feeders for supporting ex vivo expansion of umbilical cord blood derived CD34 + cells. Stem Cell Res Ther 6(1):201

15. Amiri F, Halabian R, Dehgan Harati M, Bahadori M, Mehdipour A, Mohammadi Roushandeh A et al (2015) Positive selection of Wharton's jelly-derived CD105 + cells by MACS technique and their subsequent cultivation under suspension culture condition: A simple, versatile culturing method to enhance the multipotentiality of mesenchymal stem cells. Hematology 20(4):208-216

16. Ghaneialvar H, Soltani L, Rahmani HR, Lotfi AS, Soleimani M (2018) Characterization and classification of mesenchymal stem cells in several species using surface markers for cell therapy purposes. Indian J Clin Biochem 33(1):46-52

17. Driscoll J, Patel T (2019) The mesenchymal stem cell secretome as an acellular regenerative therapy for liver disease. Journal of gastroenterology, 1-11

18. Sagaradze G, Grigorieva O, Nimiritsky P, Basalova N, Kalinina N, Akopyan Z et al (2019) Conditioned medium from human mesenchymal stromal cells: towards the clinical translation. Int J Mol Sci 20(7):1656

19. Akhter S, Rahman MM, Lee HS, Kim H-J, Hong S-T (2013) Dynamic roles of angiopoietin-like proteins $1,2,3,4,6$ and 7 in the survival and enhancement of ex vivo expansion of bone-marrow hematopoietic stem cells. Protein cell 4(3):220-230

20. Pawitan JA (2014) Prospect of stem cell conditioned medium in regenerative medicine. BioMed research international, 2014

21. Khodadi E, Shahrabi S, Shahjahani M, Azandeh S, Saki N (2016) Role of stem cell factor in the placental niche. Cell tissue research 366(3):523-531

22. Oubari F, Amirizade N, Mohammadpour H, Nakhlestani M, Zarif MN (2015) The important role of FLT3-L in ex vivo expansion of hematopoietic stem cells following co-culture with mesenchymal stem cells. Cell Journal (Yakhteh) 17(2):201

23. Farahbakhshian E, Verstegen MM, Visser TP, Kheradmandkia S, Geerts D, Arshad S et al (2014) Angiopoietin-like protein 3 promotes preservation of stemness during ex vivo expansion of murine hematopoietic stem cells. PLoS One 9:8

24. Richter J, Traver D, Willert K (2017) The role of Wnt signaling in hematopoietic stem cell development. Crit Rev Biochem Mol Biol 52(4):414-424 
25. Huang J, Nguyen-McCarty M, Hexner EO, Danet-Desnoyers G, Klein PS (2012) Maintenance of hematopoietic stem cells through regulation of Wnt and mTOR pathways. Nature medicine 18(12):1778

26. Liu FD, Tam K, Pishesha N, Poon Z, Van Vliet KJ (2018) Improving hematopoietic recovery through modeling and modulation of the mesenchymal stromal cell secretome. Stem Cell Res Ther 9(1):1-14

27. Futrega K, Atkinson K, Lott WB, Doran MR (2017) Spheroid coculture of hematopoietic stem/progenitor cells and monolayer expanded mesenchymal stem/stromal cells in polydimethylsiloxane microwells modestly improves in vitro hematopoietic stem/progenitor cell expansion. Tissue Engineering Part C: Methods 23(4):200-218

28. Huang X, Zhu B, Wang X, Xiao R, Wang C (2016) Three-dimensional co-culture of mesenchymal stromal cells and differentiated osteoblasts on human bio-derived bone scaffolds supports active multi-lineage hematopoiesis in vitro: functional implication of the biomimetic HSC niche. Int J Mol Med 38(4):1141-1151

29. Khong D, Li M, Singleton A, Chin L-Y, Parekkadan B (2018) Stromalized microreactor supports murine hematopoietic progenitor enrichment. Biomed Microdevices 20(1):13

30. Mayle A, Luo M, Jeong M, Goodell MA (2013) Flow cytometry analysis of murine hematopoietic stem cells. Cytometry Part A 83(1):27-37

31. Kiani AA, Abdi J, Halabian R, Roudkenar MH, Amirizadeh N, Soleiman Soltanpour M et al (2014) Over expression of HIF-1a in human mesenchymal stem cells increases their supportive functions for hematopoietic stem cells in an experimental co-culture model. Hematology 19(2):85-98

32. Amiri F, Halabian R, Salimian M, Shokrgozar MA, Soleimani M, Jahanian-Najafabadi A et al (2014) Induction of multipotency in umbilical cord-derived mesenchymal stem cells cultivated under suspension conditions. Cell Stress Chaperones 19(5):657-666

33. Bhang SH, Lee S, Shin J-Y, Lee T-J, Jang H-K, Kim B-S (2014) Efficacious and clinically relevant conditioned medium of human adipose-derived stem cells for therapeutic angiogenesis. Mol Ther 22(4):862-872

34. Zhao D, Liu L, Chen Q, Wang F, Li Q, Zeng Q et al (2018) Hypoxia with Wharton's jelly mesenchymal stem cell coculture maintains stemness of umbilical cord blood-derived CD34 + cells. Stem Cell Res Ther 9(1):1-11

35. Mohammadali F, Abroun S, Atashi A (2018) Mild hypoxia and human bone marrow mesenchymal stem cells synergistically enhance expansion and homing capacity of human cord blood CD34 + stem cells. Iranian journal of basic medical sciences 21(7):709

36. Antebi B, Rodriguez LA, Walker KP, Asher AM, Kamucheka RM, Alvarado L et al (2018) Short-term physiological hypoxia potentiates the therapeutic function of mesenchymal stem cells. Stem Cell Res Ther 9(1):265

37. Nowicki M, Stelmach P, Szmigielska-Kapłon A (2018) Selected factors influencing angiogenesis and hematopoietic niche. Acta Haematol Pol 49(3):112-120 
38. Mousavi SH, Abroun S, Soleimani M, Mowla SJ (2018) 3-Dimensional nano-fibre scaffold for ex vivo expansion of cord blood haematopoietic stem cells. Artificial cells nanomedicine biotechnology 46(4):740-748

39. Ahmadnejad M, Amirizadeh N, Mehrasa R, Karkhah A, Nikougoftar M, Oodi A (2017) Elevated expression of DNMT1 is associated with increased expansion and proliferation of hematopoietic stem cells co-cultured with human MSCs. Blood research 52(1):25-30

40. Martin GH, Park CY (2017) EPCR: a novel marker of cultured cord blood HSCs. Blood The Journal of the American Society of Hematology 129(25):3279-3280

41. Subramaniam A, Talkhoncheh MS, Magnusson M, Larsson J (2019) Endothelial protein C receptor (EPCR) expression marks human fetal liver hematopoietic stem cells. haematologica 104(2):e47

42. Fares I, Chagraoui J, Lehnertz B, MacRae T, Mayotte N, Tomellini E et al (2017) EPCR expression marks UM171-expanded CD34 + cord blood stem cells. Blood 129(25):3344-3351

43. Iwasaki H, Arai F, Kubota Y, Dahl M, Suda T (2010) Endothelial protein C receptor-expressing hematopoietic stem cells reside in the perisinusoidal niche in fetal liver. Blood The Journal of the American Society of Hematology 116(4):544-553

44. Zhang Y, Gao Y (2016) Novel chemical attempts at ex vivo hematopoietic stem cell expansion. Int J Hematol 103(5):519-529

45. Yaghoobi MM, Mowla SJ, Tiraihi T (2005) Nucleostemin, a coordinator of self-renewal, is expressed in rat marrow stromal cells and turns off after induction of neural differentiation. Neurosci Lett 390(2):81-86

46. Yoshida R, Fujimoto T, Kudoh S, Nagata M, Nakayama H, Shinohara M et al (2011) Nucleostemin affects the proliferation but not differentiation of oral squamous cell carcinoma cells. Cancer Sci 102(7):1418-1423

47. Hall T, Walker M, Ganuza M, Holmfeldt P, Bordas M, Kang G et al (2018) Nfix Promotes Survival of Immature Hematopoietic Cells via Regulation of c-Mpl. Stem Cells 36(6):943-950

48. Holmfeldt P, Pardieck J, Saulsberry AC, Nandakumar SK, Finkelstein D, Gray JT et al (2013) Nfix is a novel regulator of murine hematopoietic stem and progenitor cell survival. Blood The Journal of the American Society of Hematology 122(17):2987-2996

49. Ho S-Y, Ling T-Y, Lin H-Y, Liou JT-J, Liu F-C, Chen I et al (2017) SDF-1/CXCR4 signaling maintains stemness signature in mouse neural stem/progenitor cells. Stem cells international, 2017

\section{Figures}



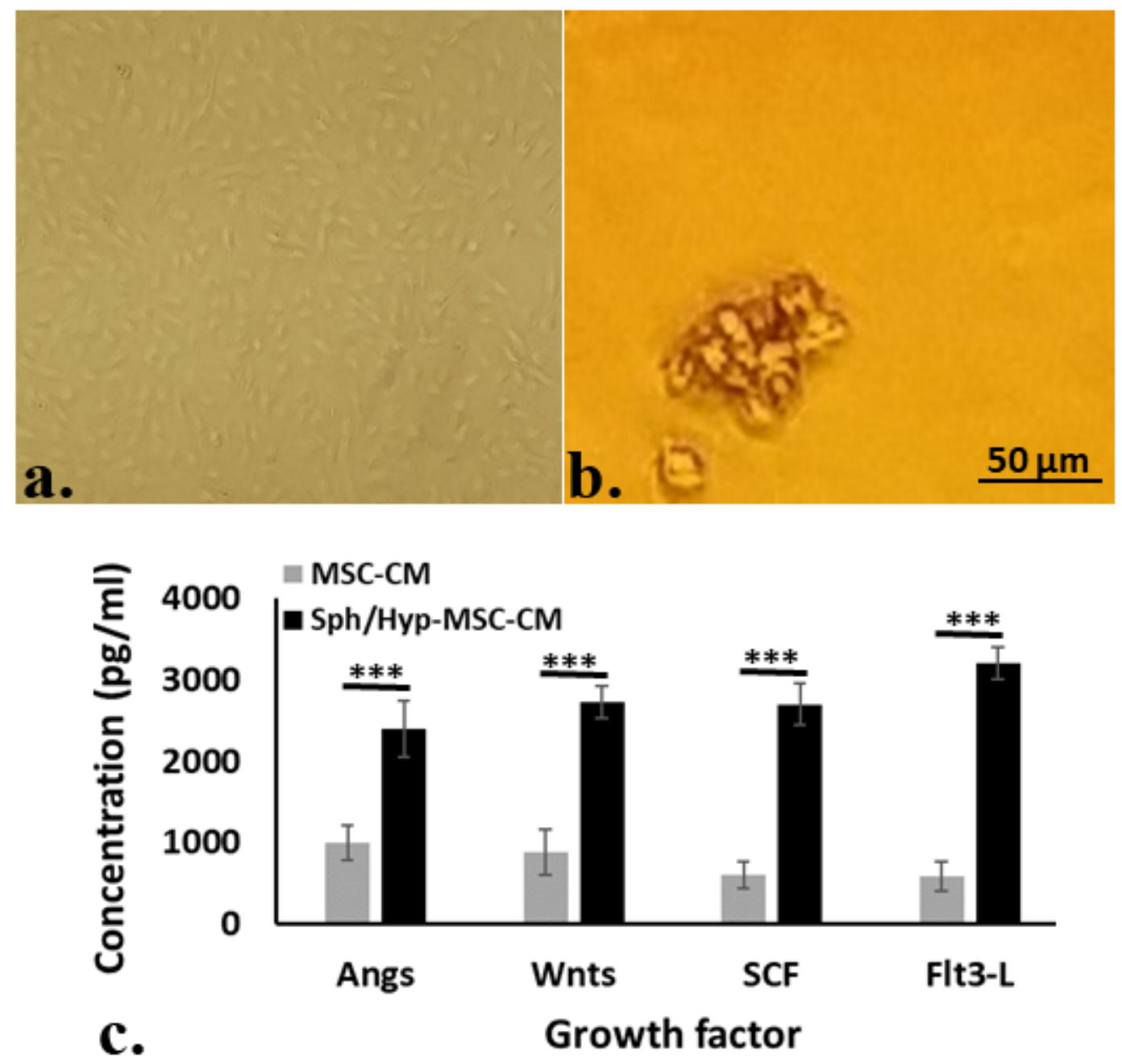

\section{Figure 1}

Isolation of WJ-MSCs and assessment of their paracrine potentialities. a. Adherent monolayer WJ-MSCs. b. Non-adherent WJ-MSC spheres in poly HEMA-coated plates. Scale bar 50 $\mathrm{mm}$. c. Evaluation of growth factor concentrations in MSC-CM and Sph/Hyp-MSC-CM with ELISA method. The Angs, Wnts, SCF, and Flt3-L concentrations were more in Sph/Hyp-MSC-CM. Data are shown as mean \pm SD. ${ }^{\star * \star P} P<0.001$ Sph/Hyp-MSC-CM vs MSC-CM. WJ-MSC: Wharton's jelly-derived-mesenchymal stem cell, Sph/Hyp-MSCCM: Conditioned medium of WJ-MSC spheres cultivated under hypoxic condition, MSC-CM: Conditioned medium of adherent WJ-MSCs, Angs: Angiopoietins, Wnts: Wingless-related integration sites, SCF: Stem cell factor, Flt3-L: Fms-like tyrosine kinase 3-Ligand. 


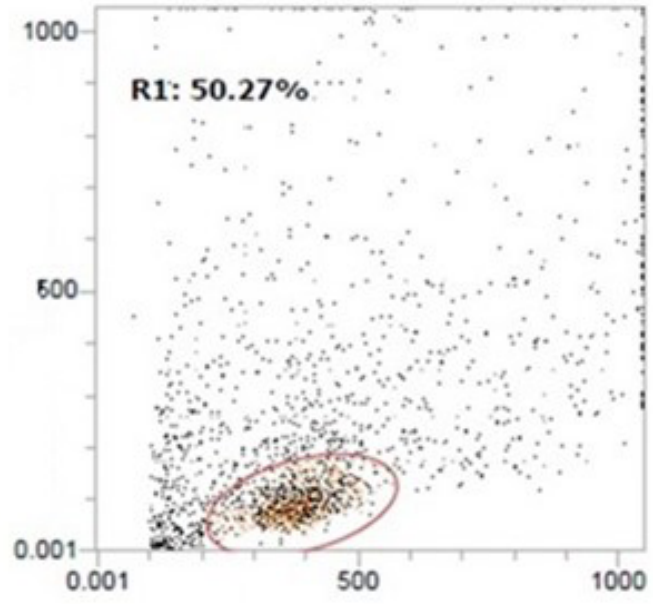

al.

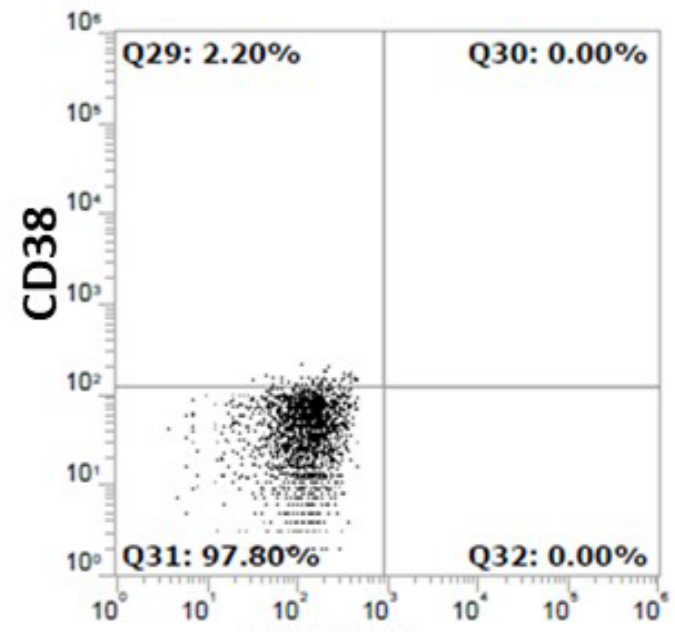

alll.

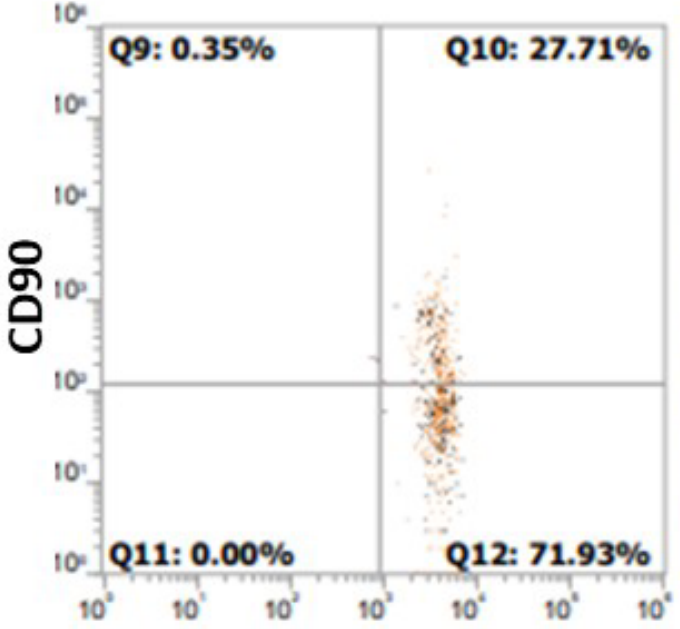

all. CD34

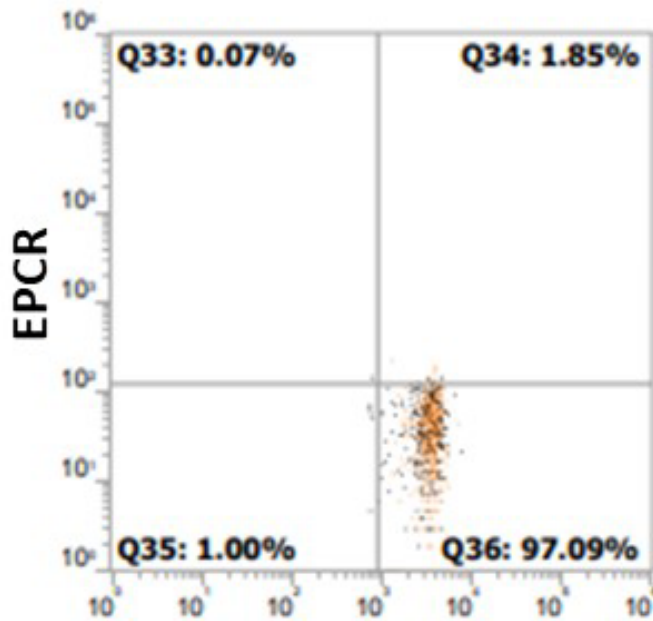

CD34

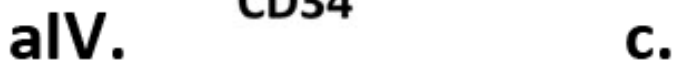

$50 \mu \mathrm{m}$

\section{Figure 2}

Isolation of hematopoietic stem cells (HSCs) and co-culturing them with adherent MSCs or MSC spheres.

a. Flowgram of isolated HSCs by magnetic-activated cell sorting. I) Cell gating, II) Expression of CD34 and CD90, III) Expression of CD38 and CD45RA, and IV) Expression of EPCR on CD34 positive cells. b. Coculturing HSCs with adherent MSCs under hypoxia condition. c. Co-culturing HSCs with MSC spheres

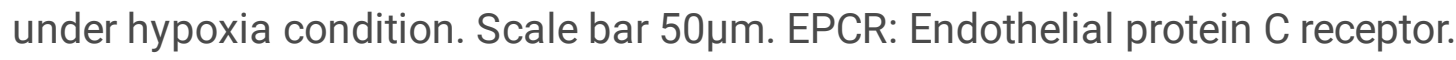



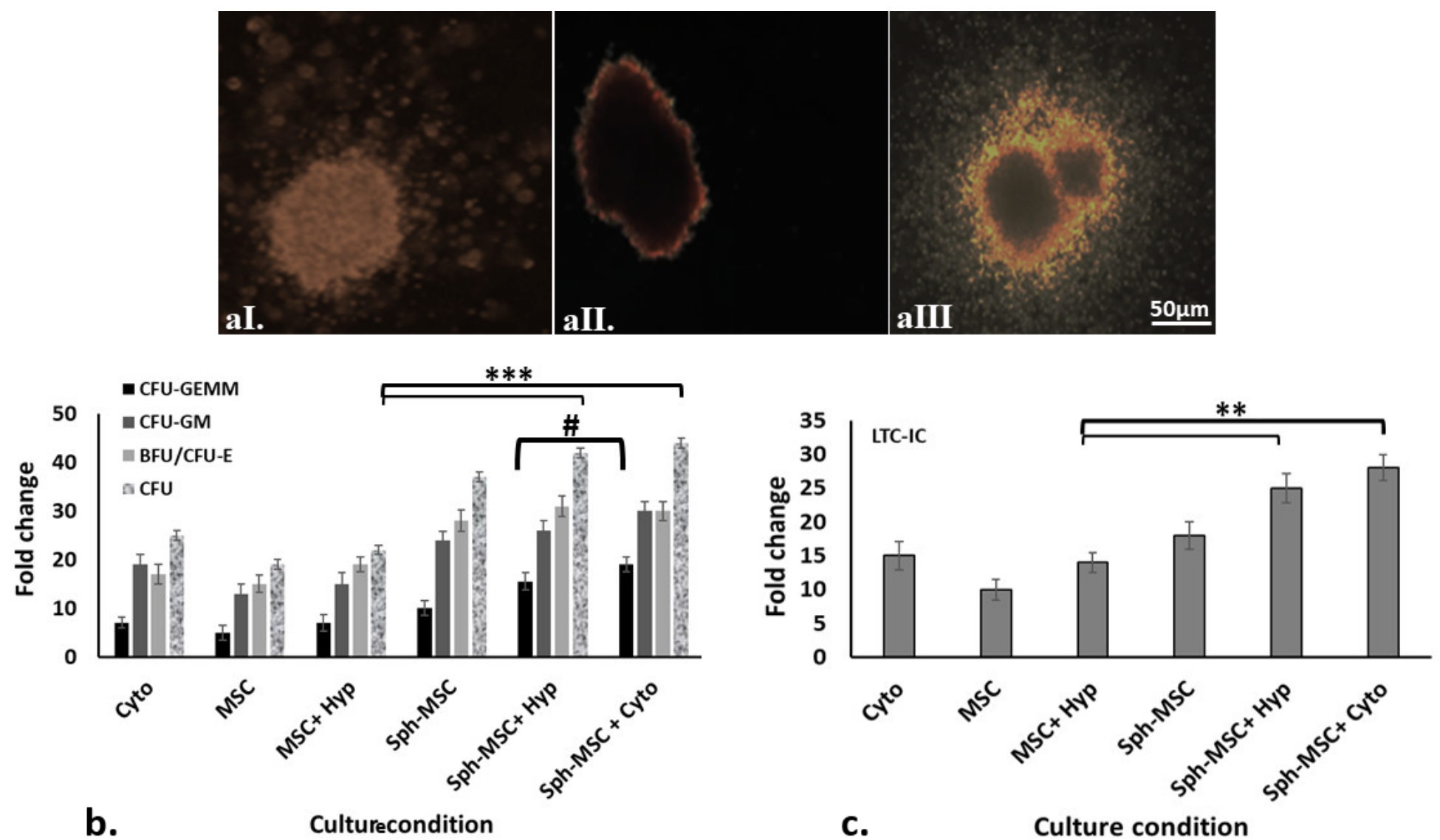

Figure 3

CFU and LTC-IC assay of expanded HSCs in different conditions on the 10th day of expansion. a. Image

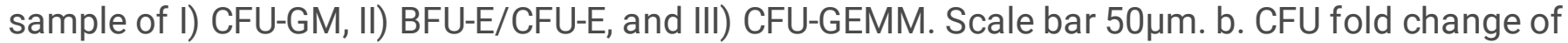
different groups. The fold change of the colonies was significantly more in Sph-MSC+ Cyto and SphMSC+ Hyp groups that were co-cultured with MSC spheres. C. The LTC-IC fold change in different conditions. The fold change of LTC-IC was higher in the Sph-MSC+ Cyto and Sph-MSC+ Hyp groups. The fold change was calculated according to fresh unexpanded cells. Data is shown as mean \pm SD.

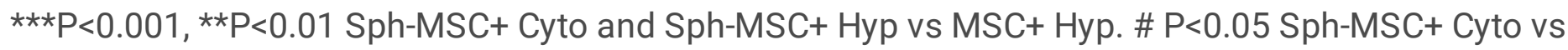
Sph-MSC+ Hyp. CFU: Colony-forming unit, LTC-IC: Long-term culture initiating cell, CFU-GM: Granulocyte macrophage-colony forming unit, BFU-E/CFU-E: Erythroid burst/colony-forming unit, CFU-GEMM: Granulocyte erythrocyte monocyte megakaryocyte-colony forming unit, Cyto: Cytokines without a feeder, MSC: Co-culturing with adherent MSCs, MSC+ Hyp: Co-culturing with adherent MSCs under hypoxic condition, Sph-MSC: Co-culturing with MSC spheres, Sph-MSC+ Hyp: Co-culturing with MSC spheres under hypoxic condition, Sph-MSC+ Cyto: Co-culturing with MSC spheres with cytokine addition. 

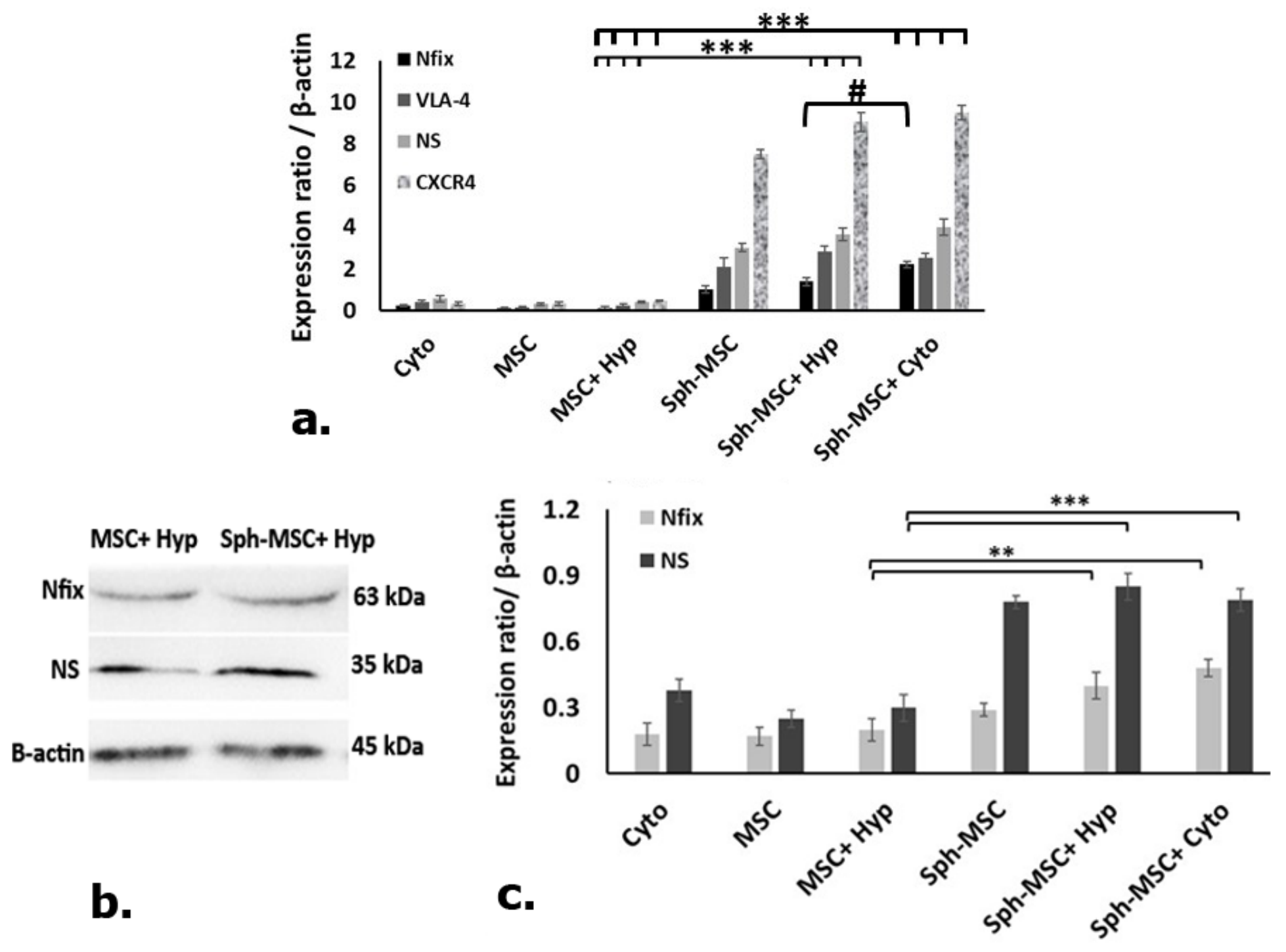

Figure 4

Evaluation of self-renewal and homing markers at transcriptional and translational levels on the 10th day of expansion. a. Real-time-PCR analysis for the expression of CXCR4, VLA-4, NS, and Nfix genes. The expression of the mentioned homing and self-renewal genes was up-regulated in the experimental groups co-cultured with MSC spheres in different conditions. b. Western blot analysis for NS and Nfix proteins. NS and Nfix, as self renewality markers, were assayed by western blotting. b. Western blot image for NS and Nfix protein in the MSC+ Hyp and Sph-MSC+ Hyp groups. c. Semi-quantification of protein the density of bands in different groups. Sph-MSC+ Cyto and Sph-MSC+ Hyp expressed more NS and Nfix proteins than MSC+ Hyp. Data are shown as mean \pm SD. ${ }^{\star \star *} P<0.001{ }^{* * P}<0.01$ Sph-MSC+ Cyto and SphMSC+ Hyp vs MSC+ Hyp, \#P<0.05 Sph-MSC+ Cyto vs Sph-MSC+ Hyp. CXCR4: C-X-C chemokine receptor type 4, VLA-4: Very late antigen-4, NS/ NS: Nucleostemin, Nfix/ Nfix: Nuclear factor I/X. Cyto: Cytokines without a feeder, MSC: Co-culturing with adherent MSCs, MSC+ Hyp: Co-culturing with adherent MSCs under hypoxic condition, Sph-MSC: Co-culturing with MSC spheres, Sph-MSC+ Hyp: Co-culturing with MSC spheres under hypoxic condition, Sph-MSC+ Cyto: Co-culturing with MSC spheres with cytokine addition. 

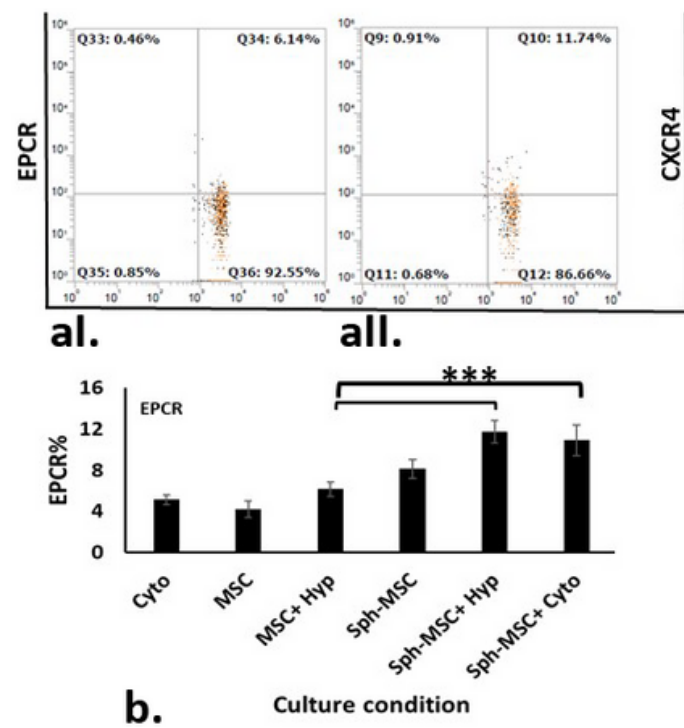

b.

Culture condition

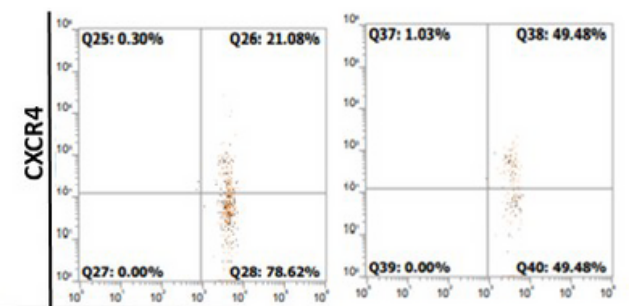

cll.

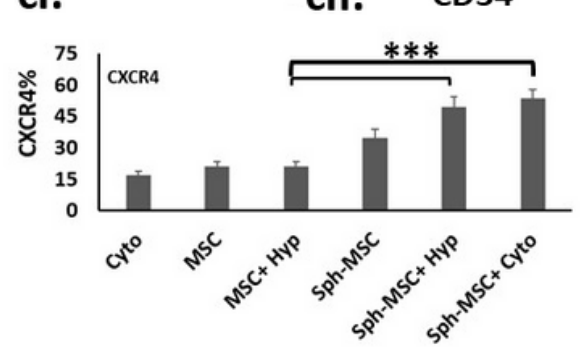

d.
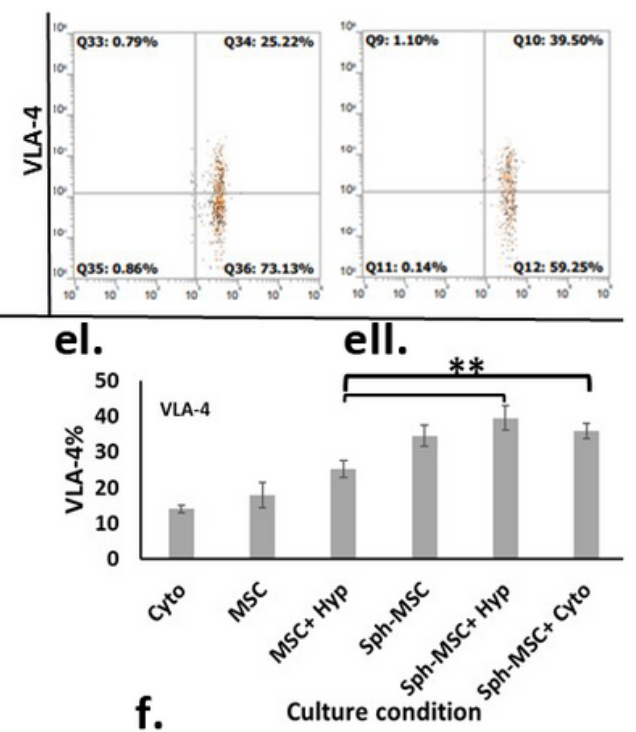

Figure 5

Flow cytometry analysis for EPCR, CXCR4, and VLA-4 on the 10th day of expansion. al. Flow cytometry graph for the expression of CD34 and EPCR in the MSC+ Hyp group. all. Flow cytometry graph for the expression of CD34 and EPCR in the Sph-MSC+ Hyp group. b. Quantification of EPCR expression in different groups. Sph-MSC+ Hyp expressed the highest percentage of EPCR. cl. Flow cytometry graph for the expression of CD34 and CXCR4 in MSC+ Hyp group. cll. Flow cytometry graph for the expression of CD34 and CXCR4 in the Sph-MSC+ Hyp group. d. Quantification of CXCR4 expression in different groups. Sph-MSC+ Hyp and Sph-MSC+ Cyto expressed the CXCR4 homing marker more than MSC+ Hyp. el. Flow cytometry graph for the expression of CD34 and VLA-4 in the MSC+ Hyp group. ell. Flow cytometry graph for the expression of CD34 and VLA-4 in Sph-MSC+ Hyp group. f. Quantification of VLA-4 expression in different groups. The expression of the VLA-4 homing marker was enhanced when co-cultured with MSC spheres in the Sph-MSC+ Hyp, and Sph-MSC+Cyto groups. Data is shown as mean $\pm S D$. ${ }^{*} * P<0.001$, **P<0.01 Sph-MSC+ Cyto and Sph-MSC vs MSC+ Hyp. CXCR4: C-X-C chemokine receptor type 4, VLA-4: Very late antigen-4. Cyto: Cytokines without a feeder, MSC: Co-culturing with adherent MSCs, MSC+ Hyp: Co-culturing with adherent MSCs under hypoxic condition, Sph-MSC: Co-culturing with MSC spheres, SphMSC+ Hyp: Co-culturing with MSC spheres under hypoxic condition, Sph-MSC+ Cyto: Co-culturing with MSC spheres with cytokine addition. 